\title{
INTEGRAL GROUP RINGS WITH TRIVIAL CENTRAL UNITS
}

\author{
JÜRGEN RITTER AND SUDARSHAN K. SEHGAL
}

(Communicated by Maurice Auslander)

\begin{abstract}
In this note finite groups $G$ whose integral group ring $\mathrm{Z} G$ has only trivial central units are classified.
\end{abstract}

In this note we classify finite groups $G$ whose integral group ring $\mathbf{Z} G$ has only trivial central units; a unit being trivial if it is of the form $\pm g, g \in G$. This question was raised by Goodaire and Parmenter [2].

It was proved by Higman ([1], [3, p. 57]) that all units of $\mathbf{Z} G$ are trivial if and only if

(a) $G$ is Abelian with exponent a divisor of 4 or 6 , or

(b) $G=K_{8} \times E$, where $K_{8}$ is the quaternion group of order 8 and $E$ is an elementary Abelian 2-group.

It follows easily that all units of a commutative group ring $\mathrm{ZG}$ are trivial if and only if:

For every $x \in G$ and every natural number $j$, relatively prime to $|G|$, we have $x^{j}=x$ or $x^{j}=x^{-1}$.

Denoting by $\sim$ conjugation in $G$, we state our result:

Theorem. Let $G$ be a finite group. All central units of $\mathbf{Z} G$ are trivial if and only if for every $x \in G$ and every natural number $j$, relatively prime to $|G|, x^{j} \sim x$ or $x^{j} \sim x^{-1}$.

Proof. At first we recall that any finite group of central units of $\mathbf{Z} G$ consists of trivial units only [4, p. 46]. It suffices to prove that the following conditions are equivalent:

(1) $\mathbf{Z} G$ has only a finite number of central units.

(2) The character field $\mathbf{Q}(\chi)$ of each absolutely irreducible character $\chi$ of $G$ is either $\mathbf{Q}$ or imaginary quadratic.

(3) For every $x \in G$ and every natural number $j$, relatively prime to $|G|$, $x^{j} \sim x$ or $x^{j} \sim x^{-1}$.

Received by the editors April 10, 1989 and, in revised form, May 19, 1989.

1980 Mathematics Subject Classification (1985 Revision). Primary 16A25, 16A26; Secondary $20 \mathrm{C} 05$.

This work is supported by NSERC Grant A-5300. 
(a) We shall first show that (1) and (2) are equivalent. Since the center of $\mathbf{Q} G$ is generated by the class sums, the center $Z$ of $\mathbf{Z} G$ is an order in the center of $\mathbf{Q} G$, the latter being the direct sum of all character fields $\mathbf{Q}(\chi)$ [3, p. 544]. Hence $Z$ is of finite additive index in the unique maximal order $\oplus_{\chi} O_{\chi}$ of $\oplus_{\chi} \mathbf{Q}(\chi)$, with $O_{\chi}$ denoting the ring of integers in $\mathbf{Q}(\chi)$. Thus the unit group of $Z$ is of finite index in the multiplicative group $\oplus_{\chi}\left(O_{\chi}\right)^{\times}$ [4, p. 49]. It follows that (1) holds precisely when $\left(O_{\chi}\right)^{\times}$is finite for all $\chi$ which by the Dirichlet unit theorem is the same as (2).

(b) We next prove that (3) implies (2). Let $\sigma$ be an automorphism of $\mathbf{Q}(\chi) / \mathbf{Q}$. Extend $\sigma$ to an automorphism $\zeta \rightarrow \zeta^{j}$ of $\mathbf{Q}(\zeta)$ where $\zeta$ is a $|G|$ th root of unity. Then $\chi^{\sigma}(g)=\chi\left(g^{j}\right)=\chi(g)$ or $\chi\left(g^{-1}\right)$ by (3). We have $\chi^{\sigma}(g)=\chi(g)$ or $\bar{\chi}(g)$. Since - commutes with $\sigma$, it follows that $\chi+\bar{\chi}=\chi^{\sigma}+\bar{\chi}^{\sigma}$. Thus $\chi^{\sigma}=\chi$ or $\bar{\chi}$ by the linear independence of irreducible characters. This implies that $\mathbf{Q}(\chi)=\mathbf{Q}$ or an imaginary quadratic field.

(c) We finally show that (2) implies (3). The proof is dual of (b). For each $g \in G$ we define a function from the irreducible characters to the complex numbers, $T(g): \operatorname{Irr}(G) \rightarrow \mathbf{C}$ by $\chi \rightarrow \chi(g)$. It follows by the orthogonality relations that these functions, one for each conjugacy class of $G$, are linearly independent. Now let $(j,|G|)=1$. Then we have an automorphism $\zeta \rightarrow$ $\zeta^{j}$ of $\mathbf{Q}(\zeta)$ where $\zeta$ is a $|G|$ th root of unity. Let $\sigma$ be the restriction of this automorphism to $\mathbf{Q}(\chi)$. Then $\chi^{\sigma}(g)=\chi(g)$ or $\chi\left(g^{-1}\right)$ by (2). Thus $T\left(g^{j}\right)+T\left(g^{-j}\right)=T(g)+T\left(g^{-1}\right)$. It follows due to the linear independence of these functions that $T\left(g^{j}\right)=T(g)$ or $T\left(g^{-1}\right)$. Thus $g^{j}$ is conjugate to $g$ or $g^{-1}$ as desired.

An easy consequence of $(2)$ is:

Corollary. If all central units of $\mathbf{Z} G$ are trivial then the same is true for $\mathbf{Z} \bar{G}, \bar{G}$ a homomorphic image of $G$.

Examples. We close with a few examples of groups satisfying the condition of the theorem.

(a) $G=S_{n}$ the symmetric group on $n$-letters. In this case all the character fields are rational $[3$, p. 538].

(b) $G$ a group of order 27 . In this case, all character fields are $\mathbf{Q}, \mathbf{Q}(w)$, $w^{3}=1$.

(c) $G=\left\langle x, y: x^{7}=1=y^{3}, x^{y}=x^{2}\right\rangle$. In this case,

$$
\mathbf{Q} G=\mathbf{Q} \oplus \mathbf{Q}(w) \oplus \mathbf{Q}(\sqrt{-7})_{3 \times 3}, \quad w^{3}=1 .
$$

Observe that $V=\mathbf{Q}(\sqrt{-7})$ is the field of index 3 in $\mathbf{Q}(\zeta), \zeta^{7}=1$, and that $V$ is a three-dimensional space over $\mathbf{Q}(\sqrt{-7})$ on which $G$ acts irreducibly by 
letting $x$ act as multiplication by $\zeta$ and $y$ by the automorphism $\zeta \rightarrow \zeta^{2}$. In this example, the character fields are $\mathbf{Q}, \mathbf{Q}(w)$ and $\mathbf{Q}(\sqrt{-7})$.

\section{ACKNOWLEDGMENT}

We thank the referee for shortening the proof of the theorem.

\section{REFERENCES}

1. G. Higman, The units of group rings, Proc. London Math. Soc. (2) 46 (1940), 231-248.

2. E. G. Goodaire and M. M. Parmenter, Units in alternative loop rings, Israel J. Math. 53 (1986), 209-216.

3. B. Huppert, Endliche Gruppen I, Springer, Berlin-Heidelberg-New York, 1967.

4. S. K. Sehgal, Topics in group rings, Marcel Dekker, New York, 1978.

Institut für MAthematik der Universität Augsburg, D-8900 Augsburg, West Germany

Department of Mathematics, University of Alberta, Edmonton, Alberta T6G 2G1 CANADA 